\title{
Anticoagulant effect of low molecular weight heparin on central venous catheters in haemodialysis patients
}

\author{
Ru-Fen $\mathrm{Li}^{1}$, Xiang-Qin $\mathrm{Cui}^{2}$ and Li-Ping $\mathrm{Xu}^{1 \text { * }}$ \\ ${ }^{1}$ Hemodialysis Room, Binzhou Medical University Hospital, Binzhou, Shandong, 256603, ${ }^{2}$ Hemodialysis Room, Binzhou City \\ Center Hospital, Binzhou, Shandong, 251700, PR China \\ *For correspondence: Email: xulpping@163.com
}

\begin{abstract}
Purpose: To analyse the effect of low molecular weight heparin on venous catheters in haemodialysis patients.

Methods: This study included 140 eligible patients who were randomly and evenly divided into two groups, viz, a study group that received low molecular weight heparin and a control group that received conventional heparin. The clinical effects and incidence of complications were compared for the two groups.

Results: No significant difference in general characteristics or the incidence of tube occlusion was detected between the two groups. The mean blood flow volume of the study and control groups were $223.50 \pm 19.10$ and $222.70 \pm 18.70 \mathrm{~mL} / \mathrm{min}$, respectively $(t=0.940, p>0.05)$, and the incidences of complications (long-term vascular secondary changes and bleeding tendency within 1 year after tube in dwelling) were lower in the study group than in the control group. After haemodialysis, the activated partial thromboplastin time and prothrombin time were shortened in the study group compared with the control group, whereas the platelet level was higher in the study group $\left(146 \pm 33 \times 10^{9} / \mathrm{L}\right)$ than in the control group $\left(95 \pm 36 \times 10^{9} / \mathrm{L}\right)$.

Conclusion: The use of low molecular weight heparin as an anticoagulant solution for patients undergoing haemodialysis with central venous catheters (CVCs) is less likely to induce haemodialysisassociated complications and has fewer effects on coagulation function than conventional heparin. Thus, low molecular weight heparin seems to be more suitable as an anticoagulant solution in patients with venous catheters.
\end{abstract}

Keywords: Low molecular weight heparin, Haemodialysis, Central venous catheter, Vascular access

Tropical Journal of Pharmaceutical Research is indexed by Science Citation Index (SciSearch), Scopus, International Pharmaceutical Abstract, Chemical Abstracts, Embase, Index Copernicus, EBSCO, African Index Medicus, JournalSeek, Journal Citation Reports/Science Edition, Directory of Open Access Journals (DOAJ), African Journal Online, Bioline International, Open-J-Gate and Pharmacy Abstracts

\section{INTRODUCTION}

Vascular access is a lifeline for patients undergoing haemodialysis. However, this process can only be completed successfully in patients with chronic renal insufficiency and uraemia when it is performed in a stable, reliable, and safe manner [1]. Moreover, the extensive application of venous indwelling catheters has been associated increasingly with various types of complication, among which catheter malfunction is the most severe and common $[2,3]$. Catheter malfunction reflects a significant decline of blood flow volume in the catheter, which, in turn, inhibits the application of haemodialysis and may result in catheter-related infection. Following the occurrence of catheter malfunction, intervention measures such as body position adjustment, the reverse connection of haemodialysis pipelines, and thrombolytic 
therapy are required [4]. Catheter malfunction is induced mainly by the formation of a thrombus or fibrin shell, but the underlying formation mechanisms are quite complex [5]. Therefore, the prevention of catheter malfunction is of great clinical importance, and a variety of tube-sealing solutions are typically used to achieve this goal [6].

Low molecular weight heparin, which is separated or decomposed from ordinary heparin, exerts anticoagulant effects by inhibiting the activity of Factor $\mathrm{Xa}$ in the body [7]. In the context of haemodialysis, low molecular weight heparin is characterised by strong anticoagulant effects, a low risk of bleeding, high bioavailability, simple operation procedures, and a high degree of safety. In contrast, the long-term application of ordinary heparin can increase the risks of complications such as bleeding, osteoporosis, and hyperlipaemia in patients undergoing haemodialysis [8]. In fact, most patients suffering from end-stage renal failure who have been treated with long-term maintenance haemodialysis using ordinary heparin develop hyperlipaemia [9]. Thus, to evaluate the clinical value of low molecular weight heparin as a potential anticoagulant solution for patients with central venous catheters (CVCs), the present study enrolled patients with chronic renal insufficiency and uraemia who had undergone haemodialysis.

\section{METHODS}

\section{Patients}

The present study included 140 patients with chronic renal insufficiency and uraemia who were treated with haemodialysis at Binzhou Medical University Hospital in Binzhou, Shandong, China, between November 2013 and May 2015. The participants were divided randomly into two groups: a study group $(n=70)$ that received low molecular weight heparin as an anticoagulant solution and a control group $(n=70)$ that received ordinary heparin as an anticoagulant solution. This study included patients who required haemodialysis to treat chronic renal insufficiency, who were at least 18 years of age, were treated once with a CVC, and who agreed to join and complete all experimental protocols. Patients who declined the questionnaire survey and telephone interview; those who had received trauma surgery during the research period; those who had received anticoagulant therapy using antithrombotic reagents such as warfarin, Plavix, and aspirin; and those who developed blood coagulation disorders, haemorrhagic disorders, and/or malignant haematological diseases were excluded from the study. This study was approved by the medical ethics committee of Binzhou Medical University Hospital (approval no. BMUH201501011XLP), and all experimental protocols followed the guidelines of the Declaration of Helsinki [10].

\section{Grouping}

The study group $(n=70)$ comprised 34 males and 36 females with a mean age of $47.22 \pm 4.33$ years and indwelling catheters for a mean of $87.23 \pm 24.56$ days. Of these 70 cases, 29 $(41.40 \%)$ indwelling tubes were temporary and $41(58.60 \%)$ were long term; 18 (25.70\%) patients had single indwelling tubes and 52 $(74.30 \%)$ had multiple indwelling tubes. Primary glomerulonephritis $(44.30 \%)$, benign nephrosclerosis $(24.30 \%)$, and diabetic nephropathy $(21.40 \%)$ were the three most incident diseases in this group. The control group $(\mathrm{n}=70)$ comprised 29 males and 41 females with a mean age of $50.36 \pm 5.05$ years and indwelling catheters for a mean of $90.41 \pm 25.13$ days. Of these 70 cases, 27 (38.60\%) indwelling tubes were temporary and $43(61.40 \%)$ were long term; $15(21.40 \%)$ patients had single indwelling tubes and $55(78.60 \%)$ had multiple indwelling tubes. Primary glomerulonephritis (40 $\%)$, benign nephrosclerosis (21.40\%), and diabetic nephropathy (20\%) were the three most incident diseases in this group. Sex, age, type of catheter, experience of tube indwelling, duration of tube indwelling, and disease condition did not differ significantly between the two groups $(p>$ 0.05); hence, the results were comparable.

\section{Baseline data collection}

After obtaining informed consent from the patients, general data regarding sex, age, experience of tube indwelling, tube indwelling mode, and disease condition were collected using a questionnaire.

\section{Treatment protocol}

In the study group, low molecular weight heparin $(12.50 \mathrm{mg} / \mathrm{mL})$ was applied as the anticoagulant solution. Following haemodialysis, a sodium chloride solution was pushed rapidly into the haemodialysis tube to cleanse away residual blood, and $1-3 \mathrm{~mL}$ heparin was then injected into the arterial and venous cavity to seal the tube. The catheter clip was closed after tube sealing, a one-off heparin cap was used to seal the tube mouth, and sterile gauze was used to cover the catheter and then fixed on. In the control group, ordinary heparin $(12.50 \mathrm{mg} / \mathrm{mL})$ 
was used as the tube sealing solution and the procedure was the same as in the study group.

\section{Assessment indices}

\section{Parameters assessed during haemodialysis}

Catheter occlusion was classified into four grades: grade 0 , smooth extraction of blood and injection of solution; grade I, resistance when an empty $10-20 \mathrm{~mL}$ needle was used, but not when an empty $1 \mathrm{~mL}$ needle was used; grade II, no blood return during blood extraction, resistance when an empty $1 \mathrm{~mL}$ needle was used, and smoothing of blood vessels after the application of urokinase thrombolysis; and grade III, no blood return during blood extraction and continued blockage of blood vessels after the application of urokinase thrombolysis. In addition, blood flow volume inside the tube during haemodialysis was observed and recorded.

\section{Parameters assessed within 1 year after haemodialysis}

First, secondary changes in blood vessels after tube indwelling were assessed; these changes included the development of mural thrombus, intraductal thrombus, a fibrin shell, intima thickening at the puncture site, and/or obvious venous stenosis. In addition, bleeding tendency within 1 year after haemodialysis was assessed in terms of whether patients exhibited subcutaneous haemorrhage, upper gastrointestinal haemorrhage, nasal bleeding, and/or cerebral haemorrhage.

\section{Parameters related to metabolic functions}

Coagulation-associated indices, including activated partial thromboplastin time (APTT); prothrombin time (PT); and platelet (PLT), haemoglobin $B(\mathrm{HB})$, and haematocrit (HCT) levels, were also assessed. APTT was measured before and after haemodialysis using a normal range of $28-49 \mathrm{~s}$; the normal range for PT was $11-16 \mathrm{~s}$, and the HB and HCT levels were measured 12 months after haemodialysis.

\section{Statistical analysis}

All results are presented as means \pm standard deviations and were analysed using chi-squared $\left(x^{2}\right)$ tests or two-sided t tests with SPSS 20.0 software. $P<0.05$ were considered to indicate statistical significance.

\section{RESULTS}

\section{Comparisons of treatment-related indices between the two groups}

The tube plugging rates in the study and control groups were $22.90 \%$ and $24.30 \%$, respectively; this difference was not significant $\left(x^{2}=2.786, p>\right.$ $0.05)$. The average blood flow volumes in the study and control groups were $223.50 \pm 19.10$ $\mathrm{mL} / \mathrm{min}$ and $222.70 \pm 18.70 \mathrm{~mL} / \mathrm{min}$, respectively; this difference was not significant $(\mathrm{t}=0.940, p$ > 0.05 ; Tables 1 and 2).

\section{Long-term complications within 1 year after treatment}

Patients in both groups exhibited secondary changes, such as intima thickening at the puncture site and the development of a fibrin shell, mural thrombus, intraductal thrombus, and severe venous stenosis. Although the incidences of these secondary changes did not differ between the two groups $(p>0.05)$, the overall incidence of secondary changes was significantly lower in the study group $(7 / 70,10 \%)$ than in the control group (22/70, $31.40 \% ; p<0.05)$. The incidences of fibrin shells and intraductal thrombi (both $2.90 \%$ ) were highest in the study group, and the incidence of fibrin shells was highest in the control group (11.40\%; Table 3).

Table 1: Occurrence of tube occlusion $(\mathrm{N}=70)$

\begin{tabular}{lccccc}
\hline \multirow{2}{*}{ Variable } & \multicolumn{4}{c}{ Tube occlusion } & Tube occlusion \\
\cline { 2 - 5 } & Grade 0 & Grade I & Grade II & Grade III & rate \\
\hline Study group & 54 & 7 & 5 & 4 & $22.90 \%$ \\
Control group & 53 & 5 & 3 & 9 & $24.30 \%$ \\
$X^{2}$ & & & & & 2.786 \\
$p$ & & & & & 0.341 \\
\hline
\end{tabular}

Table 2: Blood flow rate (mean \pm SD)

\begin{tabular}{lcccc}
\hline Variable & Study group & Control group & $\mathbf{t}$ & $\boldsymbol{P}$-value \\
\hline $\begin{array}{l}\text { Mean blood flow rate } \\
(\mathrm{mL} / \mathrm{min})\end{array}$ & $223.50 \pm 19.10$ & $222.70 \pm 18.70$ & 0.940 & 0.076 \\
\hline
\end{tabular}


Table 3: Comparison of secondary changes in blood vessels after tube indwelling [ $n$, \%]

\begin{tabular}{lcccc}
\hline Variable & $\begin{array}{c}\text { Study group } \\
(\boldsymbol{n}=\mathbf{7 0})\end{array}$ & $\begin{array}{c}\text { Control group } \\
(\boldsymbol{n}=\mathbf{7 0})\end{array}$ & $\mathbf{X}^{\mathbf{2}}$ & $\boldsymbol{P}$-value \\
\hline Intima thickening of puncture & $1(1.40)$ & $4(5.70)$ & 1.574 & 0.210 \\
site & $2(2.90)$ & $8(11.40)$ & 3.244 & 0.072 \\
Fibrin shell & $1(1.40)$ & $3(4.30)$ & 0.622 & 0.430 \\
Mural thrombus & $2(2.90)$ & $2(2.90)$ & 1.524 & 0.228 \\
Intraductal thrombus & $1(1.40)$ & $5(7.10)$ & 2.681 & 0.102 \\
Severe venous stenosis & $7(10)$ & $22(31.40)$ & 10.727 & 0.001 \\
Total & & & & \\
\hline
\end{tabular}

Subcutaneous haemorrhage, upper between the two groups $(p>0.05)$. After gastrointestinal haemorrhage, nasal haemodialysis, the APTT and PT values were haemorrhage, and cerebral haemorrhage lower in the study group than in the control group occurred in both groups after haemodialysis. The $(p<0.05)$, which suggests that APTT and PT incidences of upper gastrointestinal exhibited less obvious extension in the study haemorrhage, nasal bleeding, and cerebral group. The PLT level was higher in the study haemorrhage did not differ between the groups group than in the control group $(p<0.05)$, which $(p>0.05)$, but the incidence of subcutaneous indicates that a smaller reduction in PLT level haemorrhage was significantly lower in the study occurred in the study group. The HB and HCT group than in the control group $(p<0.05)$. In addition, the overall incidence of bleeding tendency was lower in the study group than in the control group $(p<0.05)$. The incidence of nasal haemorrhage was highest $(8.60 \%)$ in the study group, and the incidence of subcutaneous haemorrhage was highest in the control group (25.70\%; Table 4).

\section{Effect of two different tube sealing solutions on coagulation in patients undergoing haemodialysis}

Prior to haemodialysis, the APTT, PT, and PLT, levels did not differ significantly between the two groups after treatment ( $p>0.05$; Table 5$)$.

\section{DISCUSSION}

In recent years, the number of patients undergoing haemodialysis has increased as the incidences of chronic diseases, such as diabetes and nephropathy, have risen among elderly patients $[11,12]$. In fact, one study showed that $54.80 \%$ of people over 65 years of age undergo haemodialysis and that diabetic nephropathy is the leading cause for this procedure $[13,14]$.

$\mathrm{HB}$, and HCT levels did not differ significantly

Table 4: Comparison of bleeding incidences between the two groups [n, \%]

\begin{tabular}{lcccc}
\hline Variable & Study group $(\boldsymbol{n = 7 0 )}$ & $\begin{array}{c}\text { Control group } \\
(\boldsymbol{n}=\mathbf{7 0})\end{array}$ & $\mathbf{X}^{\mathbf{2}}$ & $\boldsymbol{P}$-value \\
\hline Subcutaneous hemorrhage & $6(8.60)$ & $18(25.70)$ & 9.474 & 0.002 \\
Upper gastrointestinal & $3(4.30)$ & $6(8.60)$ & 1.078 & 0.299 \\
hemorrhage & $4(5.70)$ & $8(11.40)$ & 1.729 & 0.189 \\
Nasal hemorrhage & $2(2.90)$ & $4(5.70)$ & 1.273 & 0.259 \\
Cerebral hemorrhage & $15(21.40)$ & $36(51.40)$ & 17.846 & 0.000 \\
Total & & & & \\
\hline
\end{tabular}

Table 5: Changes in coagulation function-related indices before and after treatment (mean \pm SD)

\begin{tabular}{|c|c|c|c|c|c|}
\hline Variable & Time & Study group & Control group & $t$ & $P$-value \\
\hline \multirow{2}{*}{ APTT } & Before & $32.91 \pm 4.53$ & $34.67 \pm 7.86$ & 1.317 & 0.546 \\
\hline & After & $68.35 \pm 1.64$ & $79.64 \pm 11.41$ & 6.317 & 0.000 \\
\hline \multirow{2}{*}{ PT } & Before & $13.67 \pm 3.23$ & $14.76 \pm 4.56$ & 1.870 & 0.651 \\
\hline & After & $13.69 \pm 4.18$ & $19.63 \pm 5.46$ & 6.768 & 0.000 \\
\hline \multirow{2}{*}{$\operatorname{PLT}\left(\times 10^{9} / \mathrm{L}\right)$} & Before & $151 \pm 35$ & $153 \pm 32$ & 1.563 & 0.622 \\
\hline & After & $146 \pm 33$ & $95 \pm 36$ & 7.012 & 0.000 \\
\hline \multirow{2}{*}{$\mathrm{HB}(\mathrm{g} / \mathrm{L})$} & Before & $78.21 \pm 11.5$ & $75.70 \pm 12.30$ & 1.873 & 0.542 \\
\hline & After & $77.22 \pm 11.60$ & $78.64 \pm 14.20$ & 1.673 & 0.658 \\
\hline \multirow{2}{*}{ HCT } & Before & $29.13 \pm 2.80$ & $26.93 \pm 1.80$ & 1.531 & 0.706 \\
\hline & After & $29.88 \pm 2.3$ & $30.42 \pm 1.9$ & 1.499 & 0.751 \\
\hline
\end{tabular}

Abbreviations: APTT: activated partial thromboplastin time; PT: prothrombin time; PLT: platelet; HB: haemoglobin $B$; HCT: haematocrit 
Vascular access, which is considered to be a lifeline for patients undergoing haemodialysis, determines the long-term survival rate of patients to a large extent. Central venous catheterisation is an important component of vascular access during haemodialysis and, the use of an indwelling tube in the jugular or femoral vein is currently common [15]. However, the long-term indwelling of a CVC can easily result in the development of a thrombus or fibrin shell and cause poor blood flow inside the tube, which, in turn, shortens the application time [16]. To reduce the formation of intraductal thrombi and prolong the application time, the tube must be kept unobstructed; heparin is typically used as an anticoagulant in these situations. However, excessive doses of heparin may result in complications such as spontaneous haemorrhage or hyperlipidaemia [17].

A recent study showed that heparin has different influences at different concentrations; a low concentration (312 U/mL) of heparin sodium solution induces fewer adverse reactions when used for an extended period of time [18]. Low molecular weight heparin refers to heparin with a molecular diameter $<7 \times 10^{3} \mathrm{pm}$ and molecular weight between $4.0 \times 10^{3}$ and $6.5 \times 10^{3} \mathrm{D}$ in the clinic [19]. Low molecular weight heparin has multiple advantages over ordinary heparin [20]. For example, it has a small molecular diameter, high bioavailability, and less specific binding with proteins; its dose determination does not rely on quality, and its removal effects do not rely on dose. In addition, the anticoagulant Factor $\mathrm{Xa}$ activity/antithrombin activity value of ordinary heparin is approximately 1.0 , whereas that of low molecular weight heparin is $1.5-4.0$. Thus, low molecular weight heparin is associated with strong anticoagulant factor $\mathrm{Xa}$ activity and distinct antithrombotic and bleeding induction effects.

The present study showed that the overall incidences of secondary vascular changes and bleeding tendencies were lower in the study group than in the control group, which indicates that low molecular weight heparin was associated with fewer complications than was ordinary heparin. In addition, the changes in the coagulation function indices demonstrated that APTT and PT were shorter in the study group than in the control group, which indicates that these values were less extended by low molecular weight heparin. The PLT level was higher in the study group than in the control group, which indicates that the reduction in PLT was attenuated in the study group, possibly due to the unique properties of low molecular weight heparin. The effects of low molecular weight heparin on coagulation functions were also less apparent, even in patients undergoing haemodialysis for an extended period of time.

In the present study, the tube plugging rates in the study and control groups were $22.90 \%$ and $24.30 \%$, respectively; this difference was not significant, which is consistent with the results of Hao et al [21]. Moreover, the blood flow volume did not differ significantly between the study and control groups, which is in agreement with the findings of Chen et al [22] and suggests that both low molecular weight heparin and ordinary heparin can be used as anticoagulant solutions in patients with CVCs.

\section{Limitation of the study}

The present study investigated whether tube occlusion, blood flow volume, the incidence of complications, and/or coagulation function differed between patients who received low molecular weight heparin and those who received ordinary heparin as an anticoagulant. However, this study had a short follow-up period and a relatively small sample, which constrains the generalisability of the research results. In addition, the present study did not assess differences in lipid metabolism between the study and control groups.

\section{CONCLUSION}

The findings of the present study show that the use of low molecular weight heparin as an anticoagulant solution for patients with CVCs reduces the risk of tube occlusion and extends the time of catheter indwelling without increasing the risk of bleeding. Thus, the use of low molecular weight heparin for tube sealing may be suitable in clinical haemodialysis centres.

\section{DECLARATIONS}

\section{Acknowledgement}

The authors wish to thank all those who supported this work.

\section{Conflict of Interest}

No conflict of interest associated with this work.

\section{Contribution of Authors}

The authors declare that this work was done by the authors named in this article and all liabilities 
pertaining to claims relating to the content of this article will be borne by them.

\section{Open Access}

This is an Open Access article that uses a funding model which does not charge readers or their institutions for access and distributed under the terms of the Creative Commons Attribution License (http://creativecommons.org/licenses/by 14.0) and the Budapest Open Access Initiative (http://www.budapestopenaccessinitiative.org/rea d), which permit unrestricted use, distribution, and reproduction in any medium, provided the original work is properly credited.

\section{REFERENCES}

1. Hilleman D, Campbell J. Efficacy, safety, and cost of thrombolytic agents for the management of dysfunctional hemodialysis catheters: $A$ systematic review. Pharmacology 2011; 31(10): 1031-1040.

2. Guo YC, Zhang L. A retrospective analysis about the effect of low molecular weight heparin on lipid metabolism in hemeodialysis patients. Chin J Blood Purif 2003; 2(6): 311-313.

3. Wang YZ, Le MC, Li P. Effect of antimicrobialimpregnated and heparin-bonded on preventing central venous catheters infections. Chin Mod Doct 2013; 51(19): 137-139.

4. Huang XT. Complications of heparin therapy: a contemporary clinical approach to prevention and treatment. Chin J Pract Surg 2011; 31(12): 1100-1101.

5. Moran J, Sun S, Khababa I, Pedan A, Doss S, Schille, B. A Randomized Trial Comparing Gentamicin/Citrate and Heparin Locks for Central Venous Catheters in Maintenance Hemodialysis Patients. Am J Kidney Dis 2012; 59(1): 102-107.

6. Cui WP, Du B, Zhou WH, Xu F, Xu ZG, Miao LN. Metaanalysis on feasibility of sealing central venous catheter using pure heparin in hemodialysis patients. Chin $J$ Blood Purif 2011; 1(2): 67-70.

7. Weijmer MC, van den Dorpel MA, Van de Ven PJG, ter Wee PM, van Geelen JA, Groeneveld JO, van Jaarsveld BC, Koopmans MG, le Poole CY, Schrander-Van der Meer AM, et al. Randomized, clinical trial comparison of trisodiumcitrate $30 \%$ and heparinas catheter locking solution in hemodialysis patients. J Am Soc Nephrol 2005; 16(9): 2769-2777.
8. Weijmemic MC, Dossenkopp YJ, Vdevondervoort FJ, Ter Wee PM. Superior antimicrobial activity of trisodiumcitrate over heparin for catheter locking. Nephrol Dial Transplant 2002; 17(12): 2189-2195.

9. Jin LZ, Cui TL, E Y, Liu F, Liu F, Tao Y, Fu P. Application of heparin with different concentrations in cuff loaded deep intravenous catheter. Chongqing Med J 2011; 40(3): 276-278

10. Declaration of Helsinki. The 59th World Medical Conference, 2008.

11. You HZ, Ding F, Xue J, Li MX, Kuang DW, Lu FM, Gu Y, Lin ST. Infectious complications of temporary haemodialysis catheters. Chin J Infect Chemother 2005; 5(5): 266-269.

12. Xiang DZ, Verbeken E, Van Lommel A, Stas M, De Wever I. Intimal hyperplasia after long-term venous catheterization. Eur Surg Res 2000; 32: 236-245.

13. Work J. Role of access surveillance and preemptive intervention. Semin Vase Surg 2011; 24(2):137-142.

14. Semba CP, Deitcher SR, Li X, Resnansky L, Tu T, MaCluskey ER. Treatments of occluded central venous catheters with alteplase: results in 1064 patients. Vase Intev Radiol 2002; 13(12): 1199-1205.

15. Ji DX. Central venous catheterization and vascular access of blood purification. Chin J Nephrol Dialysis Transplant 2002; 11: 347-348.

16. Huang XG, Li RS. The application of dual-chamber central venous catheter with heparin in hematodialysis. Proc Clin Med 2008; 17(7): 506.

17. Li XM, Hou J, Zhang XL, Wang H. Common complications induced by deep venous catheter indwelling in blood purification and the nursing. Pract $J$ Med Pharm 2011; 28(6): 520-521.

18. Santro A, Canova C, Freyrie A, Mancini E. Vascular access for hemodialysis. J Nephrol 2006; 19(3): 259264.

19. Golestaneh L, Mokrzycki MH. Vascular access in therapeutic apheresis: update 2013. J Clin Apher 2013; 28(1): 64-72

20. Swails-Climer M. Source how data can help reduces vascular access events. Nephrol News Issues 2012; 26(12): 30-32.

21. Hao $L Q$, Huang $S$, Jiang $Y H$, Cao $D$. Observation of tube sealing effect of low molecular heparin for central venous catheter. West Chin J Pharm Sci 2006; 21(6): 608-609.

22. Chen HX, Chen RG, Chen L, Chen L, Fu JZ. Comparison of effects of ordinary heparin and dalteparin for longterm tube indwelling. Chin J Integr Trad West Nephrol 2012; 13(11): 992-994. 